\title{
Complementary Decoder Based on Polarization Modulation for the SAC-OCDMA PON
}

\author{
He Chen, Shilin Xiao, Lilin Yi, Jie Shi, Pei Yang, Yi Dong, and Weisheng Hu
}

\begin{abstract}
We propose a complementary decoder based on polarization modulation for the spectrum amplitude codedoptical code division multiple access (SAC-OCDMA) passive optical network and experimentally demonstrate its decoding performance for the first time. The experiment result verifies that the decoder can achieve decoding and complementary decoding simultaneously for balanced detection in the SAC-OCDMA system. The eye diagrams and bit error rate measurement show that it can eliminate the multiple access interference at the high code weight.
\end{abstract}

Index Terms-Liquid crystals, multiple access interference (MAI), optical code division multiple access (OCDMA), passive optical network (PON), spectrum amplitude code (SAC).

\section{INTRODUCTION}

$\mathbf{S}$ PECTRUM amplitude coded-optical code division multiple access (SAC-OCDMA) is first proposed in [1]. Since SAC-OCDMA requires electronics operating at only the bit rate rather than the chip rate and enable balanced detection scheme to eliminate the MAI, it offers lower cost and larger code set than other OCDMA techniques, which is suitable for passive optical network (PON) applications, where cost and number of user are important. With the increase of users' number, multiple access inference (MAI) becomes a critical issue in SAC-OCDMA PON systems. It is known that by employing spectral encoding and balanced detection scheme, MAI can be completely suppressed. Some decoders have been employed for balanced detection in the SAC-OCDMA system, such as acoustically tunable optical filter, Fabry-Perot filter, arrayed waveguide grating (AWG), fiber Bragg gratings (FBGs) [2-11]. However, most of the above schemes require two discrete equipments in the decoding side, one is for decoding the signal and the other is for the complementary decoding, which increases the decoding complexity.

In [12], we have demonstrated a tunable encoder/decoder based on polarization modulation for the SAC-OCDMA system, which show a good coding/decoding performance.

Manuscript received August 27, 2011; revised November 3, 2011; accepted November 16, 2011. Date of publication November 29, 2011; date of current version February 8, 2012. This work was supported in part by the National Nature Science Fund of China under Grant 60972032 and Grant 61007041 and in part by the National "863" Hi-Tech Project of China under Grant 2006AA01Z251 and Grant 2007AA01Z271.

The authors are with the Department of Electronic Engineering, State Key Laboratory of Advanced Optical Communication Systems and Networks, Shanghai Jiao Tong University, Shanghai 200240, China (e-mail: gravelhe@gmail.com; slxiao@sjtu.edu.cn; lilinyi@sjtu.edu.cn; yidong@sjtu.edu.cn; wshu@sjtu.edu.cn).

Color versions of one or more of the figures in this letter are available online at http://ieeexplore.ieee.org.

Digital Object Identifier 10.1109/LPT.2011.2177519

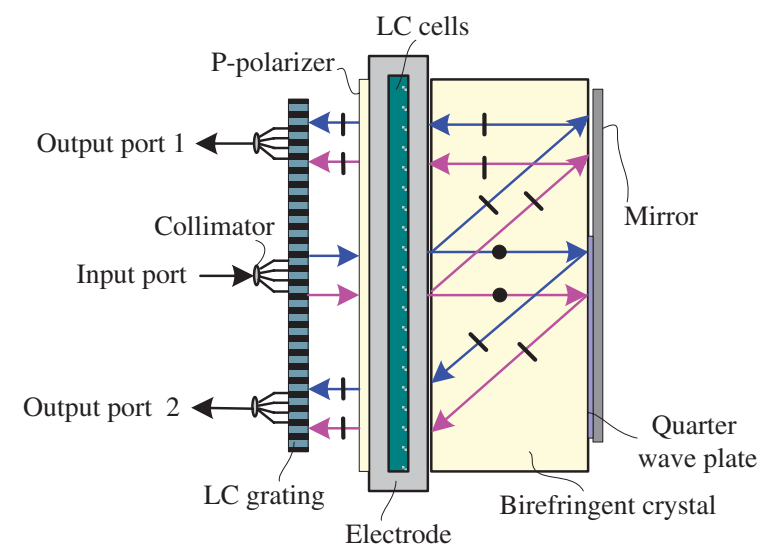

Fig. 1. Optical path of complementary decoder.

In this letter, we propose a reconfigurable decoder based on polarization modulation for the SAC-OCDMA system, which can realize decoding and complementary decoding simultaneously in the same device. The decoder is constructed with liquid crystal (LC) materials and has $40-\mathrm{dB}$ blocking ratio, and it does not block the unwanted spectral slices, but guides them to the other output port for complementary decoding. We experimentally demonstrate its decoding performance for balanced detection and prove MAI can be suppressed in SAC-OCDMA PON systems.

\section{ENCODER/DECODER BASED ON POLARIZATION MODULATION}

We employ the same device as that used in [12] for the decoder, and the optical path of the new proposed decoder is shown in Fig.1.

When the broadband optical lights from the input port are transmitted into the LC grating through a collimator, different wavelengths are separated in space (such as red line and blue line in Fig.1), and then are p-polarized after a p-polarizer. All the p-polarized wavelengths are incident on different LC cells. Every cell controlled independently by the electrode will modulate the polarization state of the individual wavelength, which will be p-polarized when the LC cells' voltage corresponding to the input port is controlled at $4 \mathrm{~V}$, and s-polarized at zero voltage. Then the birefringent crystal will guide the p-polarized wavelengths to the up-path, and the s-polarized wavelengths to the down-path through the birefriengent effect. Finally, after a mirror and LC cells, the uppath wavelengths will be directed to the output port 1, and the down-path wavelengths will be directed to the output port 2 . 


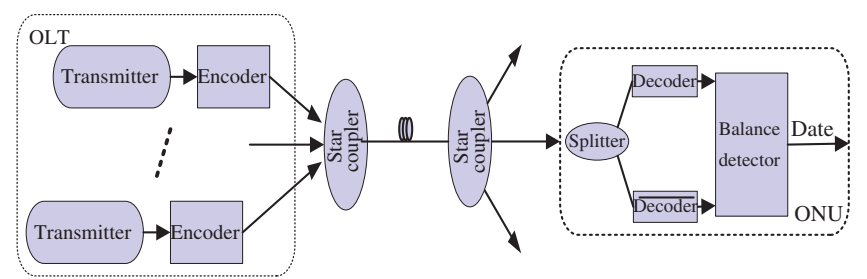

Fig. 2. Conventional balanced detection scheme.

Here a quarter wave plate is employed for changing the polarization state of the down-path wavelengths in order to make the polorazation states of the wavelength from output port 1 and output port 2 are the same. The LC cells' voltage corresponding to output port 1 and output port 2 are both controlled at above $4 \mathrm{~V}$ for minimum power loss of the spectral slices.

\section{Principle of Balance Detection Scheme}

The conventional balanced detection scheme employed in the SAC-OCDMA PON system is shown in Fig.2. The encoded signals from different transmitters in optical line terminal (OLT) are coupled together by the star coupler, and then transmit to different optical network units (ONUs). Inside the ONU, a 3-dB optical splitter is used to divide the encoded signals to a decoder and a complementary decoder for balanced detection to suppress the MAI, finally the destination signal is correctly received.

We propose using Walsh-Hadamard as the address code. Let $X^{k}=\left(x_{0}^{k} x_{1}^{k} \ldots \ldots x_{N-1}^{k}\right)\left(1 \leq k \leq N, N=2^{n}\right.$, and $n=$ $1,2,3 \ldots)$ be the $k$ th code of the Walsh-Hadamard code with code length of $\mathrm{N}$, then the auto correlation peak of $X^{k}$ will be $\sum_{i=0}^{N-1} x_{i}^{k} x_{i}^{k}=N / 2$, and the synchronous cross-correlation of two different code is $\sum_{i=0}^{N-1} x_{i}^{k} x_{i}^{m}=N / 4$. A balanced detection receiver with destined code of $X^{m}$ that computes:

$$
\begin{aligned}
R & =\sum_{i=0}^{N-1} x_{i}^{k} x_{i}^{m}-\sum_{i=0}^{N-1}\left(1-x_{i}^{k}\right) x_{i}^{m} \\
& =N / 4-N / 2+N / 4 \quad 1 \leq k \leq N, k \neq m \\
& =0
\end{aligned}
$$

will remove the signal coming from the interference users, so the MAI can be completely suppressed. This is true for any $k \neq m$, so we have a system that can support $\mathrm{N}$ simultaneous active users without any interference, and complete orthogonality among the users is theoretically achieved.

\section{EXPeriment of the Complementary Decoder FOR THE SAC-OCDMA PON}

In the experimental demonstration, we choose WalshHadamard code [13] as the address code. Here, the employed encoders are what we have proposed in [12]. The code length is 32 , and the code weight is 16 . The spectral slices are spaced by $0.8 \mathrm{~nm}$ from $1534.25 \mathrm{~nm}$ to $1559.79 \mathrm{~nm}$. The experiment setup schematic is shown in Fig. 3.

In the experiment, the amplified spontaneous emission (ASE) noise from an erbium doped fiber amplifier (EDFA) is used as a broadband source in C-band, and it is modulated

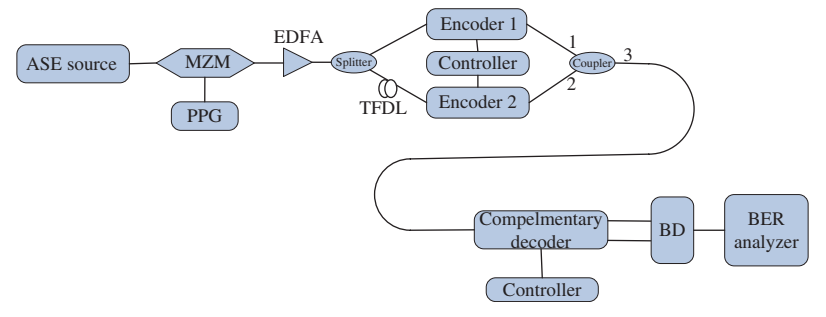

Fig. 3. Experimental setup.

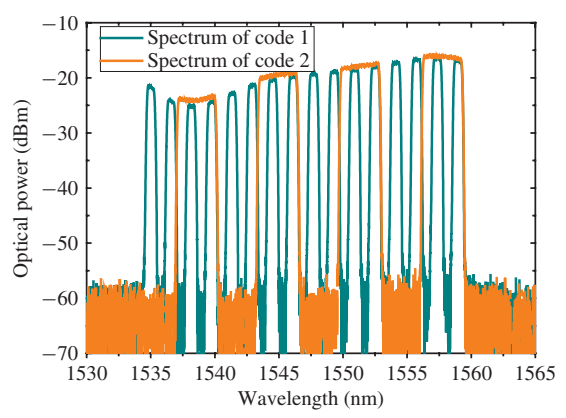

Fig. 4. Spectral slice of encoder 1 and encoder 2.

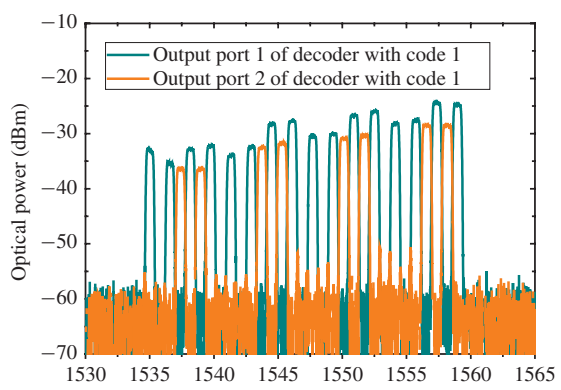

Fig. 5. Spectral slice of complentertedeygtederoder with code 1 .

by a Mach-Zehnder modulator (MZM) with a $2.5-\mathrm{Gb} / \mathrm{s}$ non return-to-zero (NRZ) pseudo-random bit sequence (PRBS) data $\left(2^{31}-1\right)$ from a pulse pattern generator (PPG). After being amplified by another EDFA, the signal is divided into two paths by a 3-dB optical splitter and then is coded by Encoder 1 and Encoder 2 respectively. A tunable fiber delay line (TFDL) is employed on the lower path to ensure the bitasynchronization of the two channels. At last, the two channels signals are coupled together by a $3-\mathrm{dB}$ coupler. At the receiver side, the signal is decoded by the complementary decoder and then detected by a balanced detector (BD). Controllers are used to tune the codes used in the encoder and decoder.

Firstly, we tune the code address of encoder 1 and encoder 2 respectively to 10101010101010101010101010101010 and 11110000111100001111000011110000 by the controller. For the Walsh-Hadamard code with code weight of 16, the number of ' 1 ' on the same position is 8 , so there are 8 same spectral slices between code 1 and code 2. Fig. 4 shows the spectral components of code 1 and code 2 .

Then we tune the code address of the complementary decoder to code 1, and their spectral components from output port 1 and output port 2 are as shown in Fig.5.

We then tune the code address of the complementary decoder to code 2, and their spectral components from output port 1 and output port 2 are shown in Fig.6. It is 


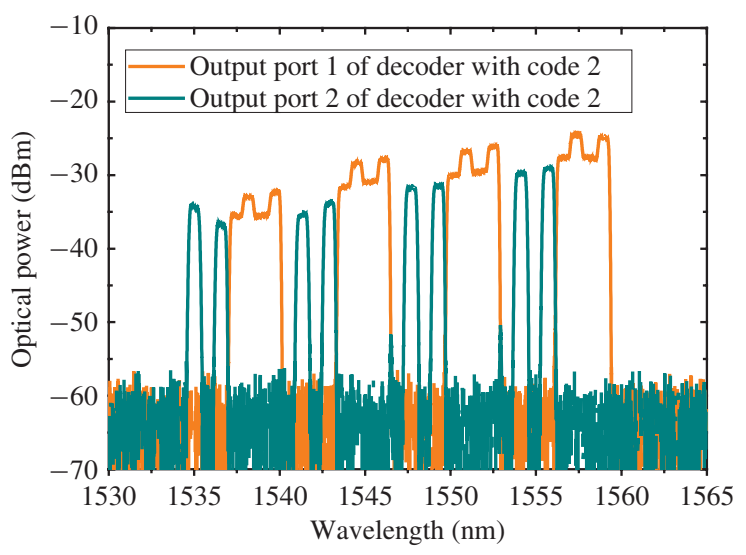

Fig. 6. Spectral slice of complementary decoder with code 2 .

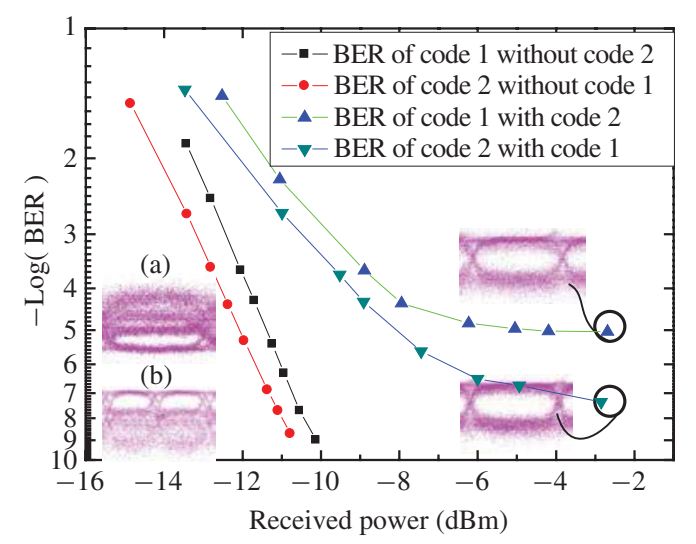

Fig. 7. BER and eye diagram of double channels.

more clearly noticed in Fig.6 that in output port 1 of the complementary decoder there are 8 interference spectral slices from the un-destined code, but output port 2 provide another 8 spectral slices for compensating the un-destined code, so after the balanced detector, the interference optical power is eliminated, and the signal is recovered correctly.

The insets (a) and (b) in Fig.7 shows the eye diagrams of code 1 and code 2 without employing the balanced detection. It is clear that the decoded signals are greatly degrade by the MAI, and the BER cannot be measured. Then the BER and eye diagram are measured under the balanced detection case, and the BER is about $10^{-6}-10^{-8}$. The BER and eye diagram of code 1 are worse than that of code 2 , and it will have the inverse result if we exchange port 1 and 2 of the coupler, so the different level of BERs between code 1 and code 2 is caused by the $3 \mathrm{~dB}$ coupler that the coupling ration is not ideal 50:50. Comparing the BER performance for a single channel case (only code 1 or code 2, i.e., no interfering user) with the BERs performance when for both channels present, the BER will get worse. Since the system has employed the balance detection scheme, most part of the MAI has been eliminated. It is induced mainly by two factors, one is the uneven broadband optical source which leads the MAI not be eliminated completely, and the other is the beat noise in SAC-OCDMA system which has been analyzed in [14].
Therefore the optical power equalization of broadband optical source and beat noise suppression are very important in the SAC-OCDMA PON system.

\section{CONCLUSION}

We have proposed a complementary decoder based on polarization modulation of LC materials for the SAC-OCDMA PON. The decoder not only has the merits of flexible reconfiguration with code addresses and high blocking ratio for the unwanted spectral slices but also achieves balanced detection scheme in one device, so one splitter is neglected and 3$\mathrm{dB}$ power budget is achieved compared with the traditional complementary decoder. The experiment verifies that it can eliminate the MAI greatly (BER decreases from $10^{-1}$ to $10^{-8}$ ), so it may be a promising option for the decoder in the SAC-OCDMA PON.

\section{REFERENCES}

[1] D. Zaccarin and M. Kavehrad, "An optical CDMA system based on spectral encoding of LED," IEEE Photon. Technol. Lett., vol. 5, no. 4, pp. 479-482, Apr. 1993.

[2] I. Hinkov, V. Hinkov, K. Iversen, and O. Ziemann, "Feasibility of optical CDMA using spectral encoding by acoustically tunable optical filters," Electron. Lett., vol. 31, no. 5, pp. 384-386, Mar. 1, 1995.

[3] K. Iversen and O. Ziemann, "An all-optical CDMA communication network by spectral encoding of LED using acoustically tunable optical filters," in Proc. URSI Int. Symp. Signals, Syst., Electron., Oct. 1995, pp. 529-532.

[4] O. Ziemann and K. Iversen, "Optical CDMA based on spectral encoding with integrated optical devices," Proc. SPIE All-Opt. Commun. Syst.: Archit., Control, Netw., vol. 2614, pp. 142-152, Oct. 1995.

[5] T. Pfeiffer, B. Deppisch, M. Kaiser, and R. Heidemann, "High speed optical network for asynchronous multiuser access applying periodic spectral coding of broadband sources," Electron. Lett., vol. 33, no. 25, pp. 2141-2142, Dec. 1, 1997.

[6] G. A. Magel, G. D. Landry, R. J. Baca, D. A. Harper, and C. A. Spillers, "Transmission of eight channels $\times 622 \mathrm{Mbit} / \mathrm{s}$ and 15 channels $\times$ $155 \mathrm{Mbit} / \mathrm{s}$ using spectral encoded optical CDMA," Electron. Lett., vol. 37, no. 21, pp. 1307-1308, Oct. 1, 2001.

[7] M. Julien, D.-P. Wei, S. Ayotte, L. A. Rusch, and S. LaRochelle, "Experimental demonstration of frequency-encoded optical CDMA using superimposed fiber Bragg gratings," in Proc. OSA/BGPP, 2003, p. 1-3, paper WD4.

[8] S. Ayotte, M. Rochette, J. Magné, L. A. Rusch, and S. LaRochelle "Experimental verification and capacity prediction of FE-OCDMA using superimposed FBG," J. Lightw. Technol., vol. 23, no. 2, pp. 724-731, Feb. 2005.

[9] J. Penon, Z. A. El-Sahn, L. A. Rusch, and S. LaRochelle, "Spectralamplitude-coded OCDMA optimized for a realistic FBG frequency response," J. Lightw. Technol., vol. 25, no. 5, pp. 1256-1263, May 2007.

[10] M. Aljada, S. Hwang, and K. Alameh, "Design of $10 \mathrm{~Gb} / \mathrm{s}$ optical encoder/decoder structure for FE-OCDMA system using SOA and optoVLSI processors," Opt. Express, vol. 16, no. 2, pp. 679-685, 2008.

[11] J. Penon, W. Mathlouthi, S. LaRochelle, and L. A. Rusch, "An innovative receiver for incoherent SAC-OCDMA enabling SOA-based noise cleaning: Experimental validation," J. Lightw. Technol., vol. 27, no. 2, pp. 108-116, Jan. 15, 2009.

[12] C. He, X. Shilin, and Y. Lilin, "A tunable encoder/decoder based on polarization modulation for the SAC-OCDMA PON," IEEE Photon. Technol. Lett., vol. 23, no. 11, pp. 748-750, Jun. 1, 2011.

[13] A. Z. G. Zahid, F. N. Hasoon, and S. Shaari, "New code structure for enhanced double weight (EDW) code for spectral amplitude coding OCDMA system," in Proc. Int. Conf. Future Comput. Commun., Apr. 2009, pp. 658-661.

[14] M. Yoshino, et al., "Beat noise mitigation of spectral amplitude coding OCDMA using heterodyne detection," IEEE Photon. Technol. Lett., vol. 26, no. 8, pp. 962-970, Apr. 15, 2008. 